\title{
Submodular Batch Selection for Training Deep Neural Networks
}

\author{
K J Joseph, Vamshi Teja R, Krishnakant Singh, Vineeth N Balasubramanian \\ Indian Institute of Technology, Hyderabad \\ \{cs17m18p100001,ee15btech11023,cs15mtech11007,vineethnb\}@iith.ac.in
}

\begin{abstract}
Mini-batch gradient descent based methods are the de facto algorithms for training neural network architectures today. We introduce a mini-batch selection strategy based on submodular function maximization. Our novel submodular formulation captures the informativeness of each sample and diversity of the whole subset. We design an efficient, greedy algorithm which can give high-quality solutions to this NP-hard combinatorial optimization problem. Our extensive experiments on standard datasets show that the deep models trained using the proposed batch selection strategy provide better generalization than Stochastic Gradient Descent as well as a popular baseline sampling strategy across different learning rates, batch sizes, and distance metrics.
\end{abstract}

\section{Introduction}

Deep learning methods are currently the state-of-the-art machine learning models for many applications, including computer vision, language understanding, and speech processing. The standard method for training deep neural networks is mini-batch stochastic gradient descent (SGD) while using backpropagation to compute the gradients. The mini-batch SGD is an optimization algorithm where mini-batches of data $D_{t}=\left\{d_{1}, d_{2}, \cdots, d_{m}\right\}$ containing $m$ examples are sampled uniformly from the dataset $D$, at time $t$. A loss function value w.r.t. the current model parameters $w_{t}$ is computed as $\mathcal{L}\left(w_{t}\right)=\sum_{i=1}^{m} l\left(d_{i} \mid w_{t}\right)$ (where $l($.$) is any differentiable loss$ function for the neural network), and the weights are updated to minimize $\mathcal{L}\left(w_{t}\right)$, according to the following equation:

$$
w_{t+1}=w_{t}-\mu_{t} \frac{\partial \mathcal{L}\left(w_{t}\right)}{\partial w_{t}}
$$

where $\mu_{t}$ is the learning rate at the $t^{t h}$ step.

In this work, we hypothesize and validate that not only is the update of $w_{t}$ given $\frac{\partial \mathcal{L}\left(w_{t}\right)}{\partial w_{t}}$ crucial, but also the selection of the mini-batch $D_{t}$ used to compute the gradient. We formulate batch selection as solving a submodular optimization problem, which contributes to significant improvement in the generalization performance of the model. To the best of our knowledge, this is the first such effort on submodular importance sampling for SGD.

Each mini-batch selection is posed as a cardinalityconstrained monotone submodular function maximization problem. This helps us leverage a greedy algorithm to solve this NP-hard combinatorial optimization problem, which guarantees a solution for a submodular objective function which is at least (in the worst case) $\left(1-\frac{1}{e}\right)$ (approximately 0.63) of the optimal solution [Nemhauser et al., 1978].

The key contribution of our work is a new submodular sampling strategy for mini-batch SGD, which helps train deep neural networks to have better generalization capability. To achieve this, we formulate a submodular objective function, which takes into account the informativeness that each sample can add to the subset and at the same time ensure that the subset as a whole, is diverse. Further, we propose an efficient algorithm to scale to high sampling rates, as required for SGD while training neural networks. We conduct extensive experimental studies of the proposed submodular minibatch selection methodology and show that it improves generalization capability of SGD as well as related previous efforts such as Loss based sampling [Loshchilov and Hutter, 2015]. We also show that the improved performance of the proposed methodology is consistent across different learning rates, mini-batch sizes and distance metrics. While submodular batch selection has been used extensively in the past for other settings such as active learning [Wei et al., 2015; Chakraborty et al., 2015] and other methods such as Determinantal Point Process (DPP) [Zhang et al., 2017] have been used to diversify minibatch selection recently, to the best of our knowledge, this is the first submodular batch selection methodology for SGD while training neural networks. Importantly, our consistent increase in generalization performance over SGD is a notable achievement, which was missing in methods proposed earlier for mini-batch selection. We use the terms batch selection and mini-batch selection interchangeably in this work.

The remainder of this paper is organized as follows. We survey the literature related to our work in Section 2; introduce our submodular function and describe our efficient implementation strategy in Section 3. The end-to-end algorithm is summarized in section 3.3. The experimental setup, main results, and ablation studies are reported in Section 4. We conclude in Section 5. 


\section{Related Work}

Mini-batch selection strategies have been explored in convex settings in the past. [Zhao and Zhang, 2014] proposed a sampling scheme based on partitioning data into balanced strata leading to faster convergence, while [Zhao and Zhang, 2015] proved that the optimal sampling distribution is directly related to the absolute values of the gradient of the samples for convex objectives. However, the prohibitive cost of evaluating the gradient impedes their usage in practice. In non-convex settings, such as in deep neural networks, there have been fewer efforts for mini-batch selection, especially in the context of SGD. Extensions of [Zhao and Zhang, 2015] to neural networks do not scale, due to the large number of trainable parameters in the deep models. Recently, [Loshchilov and Hutter, 2015; Alain et al., 2015] have tried to alleviate the cost of computing gradients by using lossbased sampling as an approximation to the gradients. Unfortunately, these methods are very sensitive to hyperparameters and perform inadequately in many cases [Katharopoulos and Fleuret, 2017]. The work was, in fact, validated only on MNIST data, which was acknowledged as a limitation of the work in [Loshchilov and Hutter, 2015]. The only other efforts to our knowledge consider more efficient approximations to the batch gradients i.e. variance of the samples [Chang et al., 2017] or an upper bound on the gradient norm [Katharopoulos and Fleuret, 2018], but the generalization performance is only comparable to SGD in most cases. On the other hand, there have been efforts to speed up mini-batch SGD in general such as [Allen-Zhu, 2017; Johnson and Zhang, 2013]. However, these efforts do not focus on batch selection or importance sampling, and show lower performance than existing importance sampling methods as noted in [Katharopoulos and Fleuret, 2018].

Submodular optimization has been successfully applied to varied tasks like document summarization [Lin and Bilmes, 2011], speech recognition systems [Wei et al., 2014], to name a few. However, there has been no work so far on using submodularity for batch selection. [Das and Kempe, 2008] proved that variance between a predictor variable using full batch and a mini-batch is submodular. We were initially motivated by this observation to propose a submodular batch selection strategy for SGD. The existing efforts that are closest to ours include Determinantal Point Process (DPP) [Zhang et al., 2017], Repulsive Point Processes (RPP) [Zhang et al., 2018] and [Singh and Balasubramanian, 2018]. Both DPP and RPP can be considered a special case of probabilistic submodular functions (PSF), although not explicitly called so in their work. However, these methods are computationally inefficient. Even with faster versions, they are prohibitively costly to be applied in deep neural networks [Li et al., 2016]. [Singh and Balasubramanian, 2018] attempt a similar objective, but the objective considered is truly not submodular, and their results are largely inconclusive. Another body of work that can be considered close to our efforts are those of selfpaced learning [Thangarasa and Taylor, 2018] and curriculum learning [Zhou and Bilmes, 2018]. However, their objectives are different, and one can consider using our batch selection strategy along with any such method too.

\section{Methodology}

\subsection{Submodular Batch Selection}

An appropriate batch selection strategy for mini-batch SGD would need to consider multiple criteria to choose the most relevant samples. A primary criterion we consider is that each selected sample must be as informative as possible. We use the model uncertainty as the measure of informativeness.

Uncertainty Score [ $\left.U\left(x_{i}\right)\right]$

The uncertainty of each data point is computed as the entropy of the current model $w^{t}$ at training iteration $t$. $C$ is the set of all classes. This allows the model to select the samples that confuses it the most in a mini-batch:

$$
U\left(x_{i}\right)=-\sum_{y \in C} P\left(y \mid x_{i}, w^{t}\right) \log P\left(y \mid x_{i}, w^{t}\right)
$$

A subset that maximizes only the Uncertainty Score, would potentially lead to the inclusion of similar data points with high entropy in the mini-batch. This redundancy should be avoided to make the mini-batch diverse. The following score helps to contain the inclusion of redundant data points:

Redundancy Score [ $\left.R\left(x_{i}\right)\right]$

Two data points $x_{i}$ and $x_{j}$ may separately furnish valuable information, but including both may make the subset less maximally informative. We use Redundancy Score to take this into account. Given $\phi($.$) to be any distance metric between the$ two data points, a greater value of the minimum distance between points in the subset would imply more diversity among the data points in the subset. (Needless to say, this score is dependent on the choice of distance metric, and we study this in our experiments.)

$$
R\left(x_{i}\right)=\min _{x_{j} \in S: i \neq j} \phi\left(x_{i}, x_{j}\right)
$$

Going further, one can notice that outlier samples may maximize the above scores. In order to counter such a selection of batches, we introduce the following score.

\section{Mean Closeness Score [ $\left.M C\left(x_{i}\right)\right]$}

This term encourages the selection of data points that are closer to the mean of all the examples $\left(\mu=\frac{1}{|V|} \sum_{k=1}^{|V|} x_{k}\right)$ to be picked. This avoids the selection of outlier samples to the extent possible.

$$
M C\left(x_{i}\right)=\phi\left(x_{i}, \mu\right)
$$

Finally, there has been recent work to show that closeness in the feature space of a deep neural network may be a better indicator of how similar two samples are (as shown by [Wei et al., 2014] in the speech domain). We hence also include a term to explicitly enforce diversity in the feature space of the given data.

\section{Feature Match Score [ $\left.F M\left(x_{i}\right)\right]$}

This score selects samples that are diverse across each dimension in the feature space. $g($.$) is a non-negative monotone$ non-decreasing concave function, $U$ is a set of fixed features 
Proceedings of the Twenty-Eighth International Joint Conference on Artificial Intelligence (IJCAI-19)

and $m_{u}\left(x_{i}\right)$ is a non-negative score, measuring the degree to which data point $x_{i}$, possesses the feature $u$.

$$
F M\left(x_{i}\right)=\sum_{u \in U} g\left(m_{u}\left(x_{i}\right)\right)
$$

Our implementation of this score (as well as others) is described in Section 4.1.

We combine the above-mentioned scores to form our objective function for batch selection, $\mathcal{F}(S)$, as below and then show the submodularity of the proposed $\mathcal{F}(S)$.

$\mathcal{F}(S)=\sum_{x_{i} \in S} \lambda_{1} U\left(x_{i}\right)+\lambda_{2} R\left(x_{i}\right)+\lambda_{3} M C\left(x_{i}\right)+\lambda_{4} F M\left(x_{i}\right)$

Given a dataset with $N$ training data points, a mini-batch of size $k$ is selected by solving the following cardinalityconstrained submodular optimization problem:

$$
\max _{S \subseteq V,|S| \leq k} \mathcal{F}(S)
$$

We now show that the score function $\mathcal{F}$ is indeed submodular and is monotonically non-decreasing. This would allow us to solve the problem in (7) using a greedy approach [Nemhauser et al., 1978].

Lemma 1. The score function $\mathcal{F}($.$) , defined in Eqn 6$ is submodular.

Proof. Consider two subsets of training examples from a dataset $V=\left\{x_{1}, x_{2}, \cdots, x_{N}\right\} ; S_{1}$ and $S_{2}$, such that $S_{1} \subseteq$ $S_{2} \subseteq V$. Let $a$ be an element not selected so far: $a \in V \backslash S_{2}$. The marginal gain of adding $a$ to $S_{1}$ is given by:

$$
\begin{aligned}
& \mathcal{F}\left(a \mid S_{1}\right)=\mathcal{F}\left(\{a\} \cup S_{1}\right)-\mathcal{F}\left(S_{1}\right) \\
& =\lambda_{1} U(a)+\lambda_{2} \min _{a_{j} \in S_{1}} \phi\left(a, a_{j}\right)+\lambda_{3} M D(a)+\lambda_{4} F M(a)
\end{aligned}
$$

Similarly, the marginal gain of adding $a$ to $S_{2}$ is given by:

$$
\begin{aligned}
& \mathcal{F}\left(a \mid S_{2}\right)=\mathcal{F}\left(\{a\} \cup S_{2}\right)-\mathcal{F}\left(S_{2}\right) \\
& \quad=\lambda_{1} U(a)+\lambda_{2} \min _{a_{j} \in S_{2}} \phi\left(a, a_{j}\right)+\lambda_{3} M D(a)+\lambda_{4} F M(a)
\end{aligned}
$$

Since $S_{1} \subseteq S_{2}$, the minimum distance of the new point $a$, from $S_{1}$ would be greater than any element from $S_{2}$, as there may exist a point in $S_{2}$ that is much closer to $a$, than any element from its subset $S_{1}$. Hence,

$$
\min _{a_{j} \in S_{1}} \phi\left(a, a_{j}\right) \geq \min _{a_{j} \in S_{2}} \phi\left(a, a_{j}\right)
$$

Thus, we can claim $\mathcal{F}\left(a \mid S_{1}\right) \geq \mathcal{F}\left(a \mid S_{2}\right)$. Hence, the score function $\mathcal{F}($.$) is submodular.$

Lemma 2. The score function $\mathcal{F}($.$) in Eqn 6$ is a monotonically non-decreasing function.

Proof. Consider a subset $S$ and an element $a \in V \backslash S$. When $a$ is added to $S$, the function value of $\mathcal{F}(\{a\} \cup S)$ changes by $\lambda_{1} U(a)+\lambda_{2} \min _{a_{j} \in S} \phi\left(a, a_{j}\right)+\lambda_{3} M D(a)+\lambda_{4} F M(a)$. All these are non-negative quantities. Thus, $\mathcal{F}(\{a\} \cup S) \geq$ $\mathcal{F}(S)$, and the score function $\mathcal{F}($.$) is hence monotonically$ non-decreasing.
Theorem 1. Let $S^{*}$ denote the optimal solution of the problem in (7) and $S$ denote the solution obtained for the same problem using a greedy approach. Then:

$$
\mathcal{F}(S) \geq\left(1-\frac{1}{e}\right) \mathcal{F}\left(S^{*}\right)
$$

Proof. Having proved that score function $\mathcal{F}($.$) is submodular$ in Lemma 1 and that it is monotonically non-decreasing in Lemma 2, the proof follows directly from Theorem 4.3 in [Nemhauser et al., 1978].

\subsection{Scaling to High Sampling Rates}

It is interesting to note that application settings where submodularity has worked well hitherto [Wei et al., 2014; 2015; Chakraborty et al., 2015; Lin and Bilmes, 2011] do not require a high sampling rate, as much as demanded by minibatch selection in SGD. Consider a mini-batch training algorithm that consists of $p$ epochs and $q$ iterations in each epoch, the submodular batch selection needs to be carried out $p \times q$ times. Concretely, for training on the CIFAR-100 dataset having 50,000 examples and a batch size of 50 ( $q$ is hence 50000 / 50), we need 100,000 batch selections for 100 epochs $(p=100)$. Even a greedy algorithm [Nemhauser $e t$ al., 1978] that uses scores such as pairwise distance metrics has a complexity of $O\left(n^{2}\right)$, where $n$ is the dataset size. This would be too slow for use with SGD. We hence seek more efficient mechanisms to implement the proposed batch selection strategy.

Recent efforts have attempted to make submodular sampling faster in a more general context (not in SGD), such as Lazy Greedy [Minoux, 1978], Lazier than Lazy Greedy (LtLG) [Mirzasoleiman et al., 2015], and Distributed Submodular Maximization [Mirzasoleiman et al., 2013]. In this work, we present a new methodology for efficient submodular sampling inspired by Distributed Submodular Maximization algorithm and Lazier than Lazy Greedy algorithm (LtLG), as described in Algorithm 1. The algorithm partitions the training set $V$ into $m$ partitions in Line 2 and runs Lazier than Lazy Greedy algorithm (LtLG) [Mirzasoleiman et al., 2015] (described later in this section) on each partition (Lines 5 through 8 ) to obtain $m$ subsets, each of size $b$. These subsets $\left(S_{i} s\right)$ are then merged in Line 9 . The final subset $S$ is selected from this merged set by running LtLG again on it. This divide-and-conquer strategy is motivated by the Distributed Submodular Maximization algorithm [Mirzasoleiman et al., 2013].

The Lazier than Lazy Greedy (LtLG) [Mirzasoleiman et al., 2015] algorithm starts with an empty set and adds an element from set $R$, which maximizes the marginal gain $\mathcal{F}(a \mid S)=\mathcal{F}(a \cup S)-\mathcal{F}(S)$. This is repeated until the cardinality constraint $(|S| \leq b)$ is met. The set $R$ is created by randomly sampling $s=\frac{|V|}{b} \log \frac{1}{\epsilon}$ items from the superset $\mathrm{V}$, where $\epsilon$ is a user-defined tolerance level. We refer the readers to [Mirzasoleiman et al., 2015] for further information. The model at the $k^{t h}$ training iteration, $w_{k}$, is used while computing $\mathcal{F}($.$) as defined in Equation 6. The solution produced by$ Algorithm 1, $S$, has the following approximation guarantee 


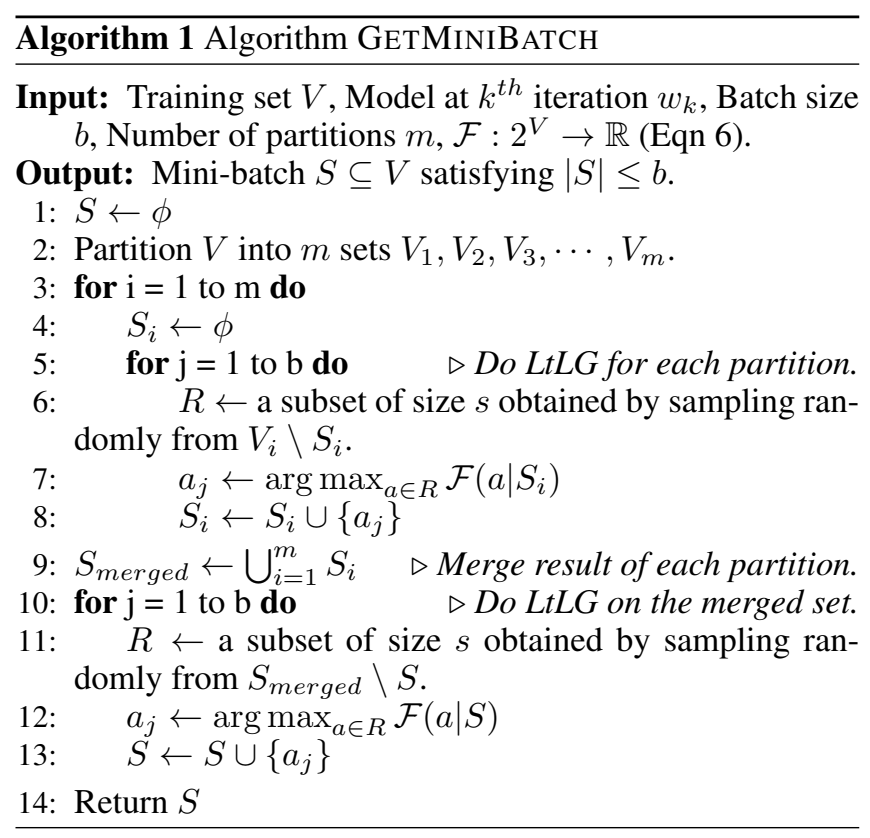

with the optimal solution $S^{*}$ :

$$
\mathcal{F}(S) \geqslant \frac{\left(1-e^{-1}\right)^{2}}{\min (m, b)}\left(1-e^{-1}-\epsilon\right) \mathcal{F}\left(S^{*}\right)
$$

Here, $m$ refers to the number of partitions, $b$ refers to the mini-batch size and $e$ is the base of natural logarithm. Our empirical results (Section 4.2) shows that the approximation is much better than this lower bound in practice.

\subsection{Gradient Descent with Submodular Batches}

The proposed batch selection strategy can work with any mini-batch gradient descent based optimization algorithms. Algorithm 2 summarizes the end-to-end training procedure.

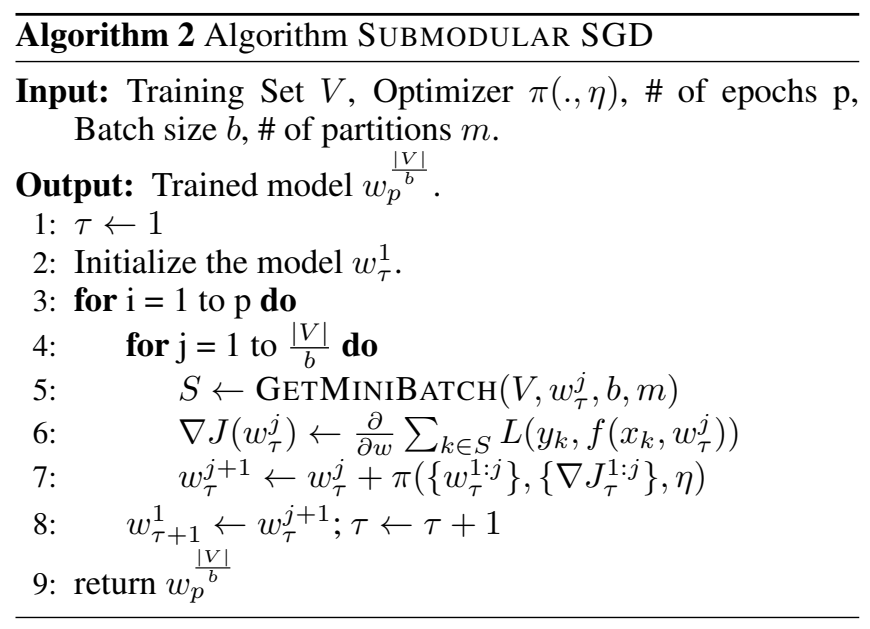

Within each iteration in each epoch, a mini-batch $S$ is selected using Algorithm 1 (Line 5). Lines 6 and 7 update $w$ with a gradient descent optimizer $\pi(., \eta)$, consuming the current set $S . \eta$ is the learning rate. Any differentiable loss function can be used as $L($.$) . Momentum-based and adaptive$ learning rate-based gradient descent methods could be used to further improve the learning based on submodular batches.

\section{Experiments and Results}

We conduct extensive experimental evaluations to study the effectiveness of submodular mini-batches in training deep neural networks over Stochastic Gradient Descent (SGD) and Loss-based sampling [Loshchilov and Hutter, 2015], as in earlier efforts such as [Katharopoulos and Fleuret, 2018]. For brevity, we refer to our proposed method of selecting submodular mini-batches for training as SubModular Data Loader (SMDL). We study the performance on the standard image classification task (as used in related earlier efforts) with SVHN [Netzer et al., 2011], CIFAR-10 and CIFAR-100 [Krizhevsky and Hinton, 2009] datasets. ResNet 20 [He et al., 2016] is used as the network architecture for SVHN and CIFAR-10, while ResNet 32 is used with CIFAR-100. Our implementation details are described in Sec 4.1, followed by the main result and various ablation study results in Sec 4.2.

\subsection{Implementation Details}

Algorithm 2 gives the generalized training procedure for submodular mini-batch selection. In each iteration, the current model $w_{\tau}^{j}$ is used to evaluate the submodular objective score (Equation 6). The feature representation for the images is obtained from the penultimate fully connected layer of this model. The probability values $\left(P\left(y \mid x_{i}, w\right)\right)$ that are used in the computation of Uncertainty Score is the softmax output from the model. Euclidean distance between the image features is used for Redundancy Score computation in Equation 3. We do an ablation study on the effect of using other distance metrics in Section 4.3. Mean Closeness Score is computed as the cosine similarity between each data-point and the mean of all the training examples.

We follow the method used in [Brahma and Othon, 2018] and [Zhou and Bilmes, 2018] to compute the fixed feature set $U$ used for evaluating Feature Match Score (Equation 5). We train a corresponding neural network, say $M$, on a random subset of training data, for an epoch. The features from the penultimate fully connected layer of $M$ is used as $U$. Square root function is used as $g(.) . m_{u}\left(x_{i}\right)$ is the feature at $u^{t h}$ index of the representation of $x_{i}$ from the model $M$.

After a grid search and an empirical study, we use the following values for the co-efficients of the terms in the objective function: $\lambda_{1}=0.2, \lambda_{2}=0.1, \lambda_{3}=0.5, \lambda_{4}=0.2$. Ablation studies of the effect of the $\lambda$ parameters are presented in Section 4.3. Each of the scores is individually normalized across the selected pool of samples, before being combined, to ensure fair contribution. All the score computation (which depends on the softmax output and the feature representation from fully-connected layers) is a function of the model at each iteration (Line 5, Algorithm 2). As we are using gradient descent for updating the parameters, we know that the model does not change drastically between iterations. Computational efficiency can be improved if we share the same model between successive iterations. We use a refresh rate of 5 for all the experiments. A study of how our method behaves with different refresh rates is shown in Figure 2(d). We note 


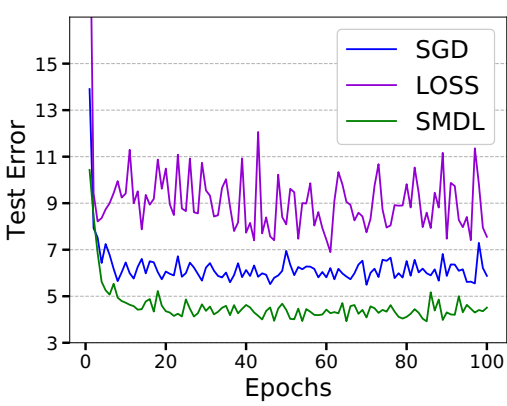

(a) Test Error on SVHN

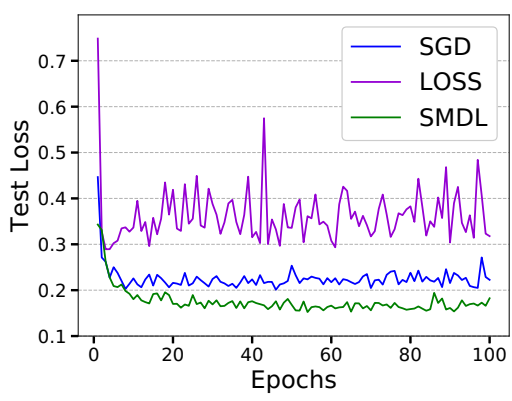

(d) Test Loss on SVHN

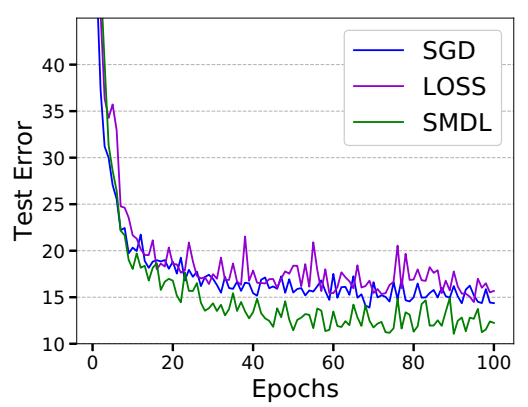

(b) Test Error on CIFAR-10

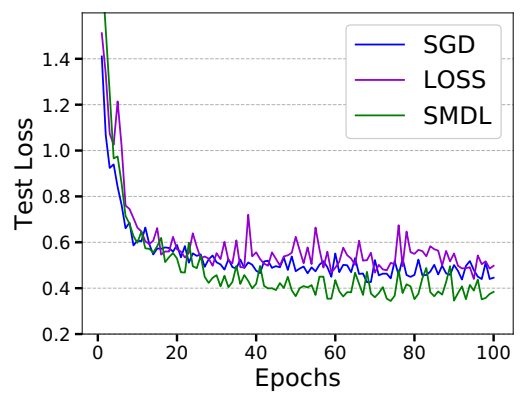

(e) Test Loss on CIFAR-10

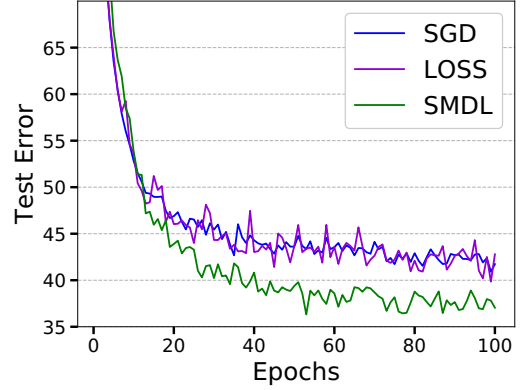

(c) Test Error on CIFAR-100

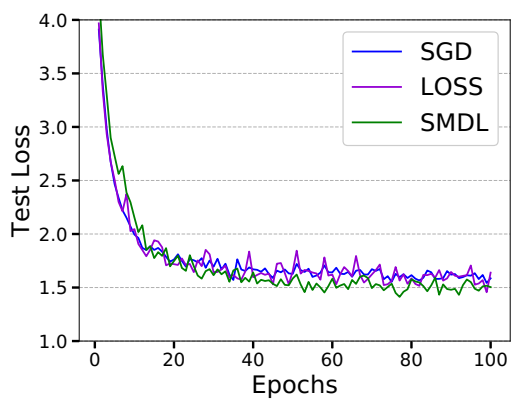

(f) Test Loss on CIFAR-100

Figure 1: Comparison of the proposed SMDL, with SGD and loss based sampling scheme on SVHN, CIFAR-10 and CIFAR-100 datasets. The test error and test loss is plotted. SMDL consistently outperforms the baselines, both in terms of loss and generalization performance.

that increasing the refresh rate decreases the performance of the model. This is because the model changes over multiple iterations, which in turn affects the quality of the mini-batch selected.

We develop a modularized and configuration-driven tool in PyTorch [Paszke et al., 2017], which implements submodular selection and the other two baseline methods: SGD and Loss based sampling [Loshchilov and Hutter, 2015]. All the experiments are run for 100 epochs with a batch size of 50, a momentum parameter of 0.9 and weight decay of 0.0001. Use of batch normalization [Ioffe and Szegedy, 2015] and adaptive learning methods like Adam [Kingma and Ba, 2014] will complement the reported results of SMDL and other baseline methods. For SGD, all the reported results are the average of five runs. The partition size ( $m$ in Algorithm 1 and 2 ) is set to 10 . Source code and supplementary material which includes additional results is available here: https://josephkj.in/projects/SMDL

\subsection{Results}

We present the major result of our proposed submodular mini-batch selection method, SMDL in Figure 1 and Table 1. We train the two network architectures on three datasets as enumerated in Section 4. The generalization performance of these classification models, as measured by their test accuracy and test loss, is used as the evaluation metric. We see from Figure 1 and Table 1 that SMDL is able to achieve lower error and loss, consistently across epochs, on all the three datasets. It is worth noting that Loss-based sampling fails significantly

\begin{tabular}{clcccc}
\hline Dataset & Method & \multicolumn{2}{c}{ Accuracy } & \multicolumn{2}{c}{ Loss } \\
\hline & & Mean & Final & Mean & Final \\
\hline \multirow{3}{*}{ SVHN } & Loss Based & 90.87 & 92.44 & 0.363 & 0.318 \\
& SGD & 93.60 & 94.34 & 0.230 & 0.215 \\
& SMDL & $\mathbf{9 5 . 4 6}$ & $\mathbf{9 5 . 4 9}$ & $\mathbf{0 . 1 7 5}$ & $\mathbf{0 . 1 8 2}$ \\
\hline \multirow{3}{*}{ CIFAR-10 } & Loss Based & 81.06 & 84.32 & 0.590 & 0.497 \\
& SGD & 82.54 & 85.63 & 0.535 & 0.445 \\
& SMDL & $\mathbf{8 4 . 5 8}$ & $\mathbf{8 7 . 7 6}$ & $\mathbf{0 . 4 8 7}$ & $\mathbf{0 . 3 8 4}$ \\
\hline \multirow{3}{*}{ CIFAR-100 } & Loss Based & 53.77 & 57.22 & 1.755 & 1.639 \\
& SGD & 53.37 & 58.27 & 1.764 & 1.586 \\
& SMDL & $\mathbf{5 7 . 2 3}$ & $\mathbf{6 2 . 9 5}$ & $\mathbf{1 . 7 1 7}$ & $\mathbf{1 . 5 0 4}$ \\
\hline
\end{tabular}

Table 1: Quantitative comparison of the proposed SMDL with SGD and Loss based sampling scheme on SVHN, CIFAR-10 and CIFAR100 datasets. Mean refers to the mean accuracy(\%) across epochs and Final refers to the final accuracy.

on SVHN. Such deterioration of generalization performance is also noted in [Loshchilov and Hutter, 2015]. The values reported on SGD are its mean after conducting five trials.

These results support our claim that selecting a mini-batch which respects diversity and informativeness of the samples helps in more generalizable deep learning models.

\subsection{Ablation Studies}

\section{Effect of Trade-off Parameters}

We study the effect of trade-off parameters that control the contribution of each of the four score functions in the submodular objective function (Equation 6). For this, we train ResNet 20 on a small subset of SVHN dataset. Except for 

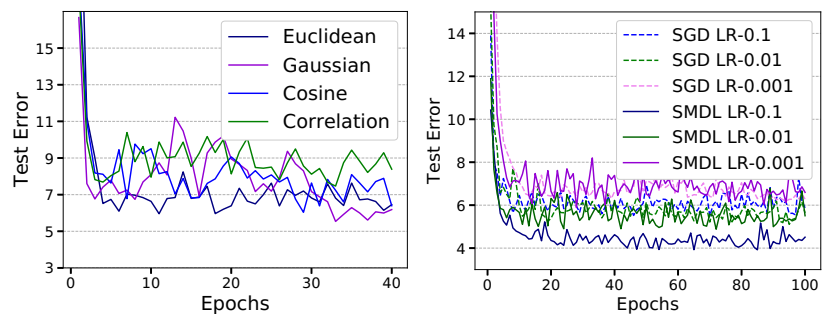

(a) Comparison with different(b) Test Error plot with different distance metrics.

learning rates (LR).
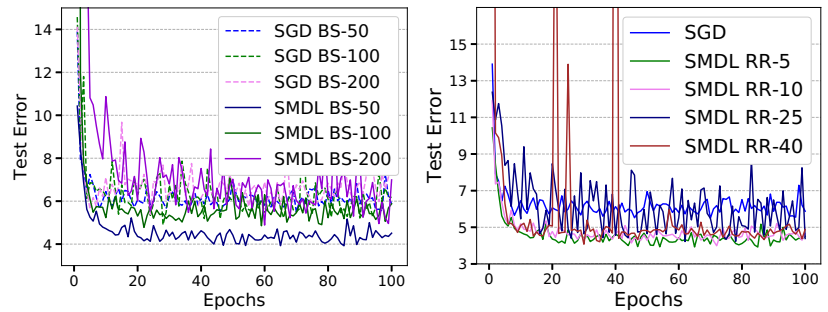

(c) Test Error plot with different(d) Test Error plot with different mini-batch sizes (BS). Refresh Rates (RR).

Figure 2: The figure shows the ablation results on SVHN dataset. 1)We find that the Euclidean distance measure performs best among other distance metrics. 2)The proposed method SMDL consistently outperforms SGD even with different batch sizes and different learning rates. We note that a batch size of 50 and a learning rate of 0.1 gives the least error for SMDL. 3) We find that a refresh rate of 5 gives the best performance. (refer Section 4.1).

the Redundancy Score (controlled via $\lambda_{2}$ ), all the other terms are modular. Hence $\lambda_{2}$ should be non-zero to make the objective function submodular. We vary each of the other $\lambda_{i}(i \in\{1,3,4\})$ with values from $\{0,0.2,0.5,0.8,1.0\}$, by fixing $\lambda_{2}=0.5$ and rest to zero. These results are reported in column 3, 5 and 6 of Table 2. Then, we fix the best values obtained for $\lambda_{1}, \lambda_{3}$ and $\lambda_{4}$ and vary $\lambda_{2}$. The result is populated in the fourth column of Table 2 . It is evident from the table that the following trade-off parameters (after normalization) achieves best performance on the subset: $\lambda_{1}=0.25, \lambda_{2}=0.25, \lambda_{3}=0.4, \lambda_{4}=0.1$. Empirically, we find that $\lambda_{1}=0.2, \lambda_{2}=0.1, \lambda_{3}=0.5, \lambda_{4}=0.2$, achieves best performance on the whole dataset. These set of trade-off parameters also generalises well to CIFAR-10 and CIFAR100 datasets.

From Table 2 we can observe that each of the score function has a profound effect on the quality of the model being trained. Setting each of them to zero hurts the performance the most (Row 1 and 2). The fourth column reveals that the submodular term has the maximal impact if we set it zero.

\section{Variations Across Different Distance Metrics}

We perform an ablation study on the impact of different distance metrics while computing the value of the submodular objective function. We choose four distance metrics and evaluate its impact on training an image classifier on SVHN dataset. Assuming $u$ and $v$ as the two vectors, the distance

\begin{tabular}{l|l|llll}
\hline \multicolumn{2}{c}{} & \multicolumn{1}{c}{$\lambda_{1}$} & $\lambda_{2}$ & $\lambda_{3}$ & $\lambda_{4}$ \\
\hline \multirow{2}{*}{0} & Mean & 64.41 & 46.17 & 64.41 & 64.41 \\
& Final & 87.91 & 83.62 & 87.91 & 87.91 \\
0.2 & Mean & 58.60 & 64.55 & 62.90 & 62.25 \\
& Final & 91.13 & 91.34 & 91.01 & $\mathbf{9 2 . 1 4}$ \\
& Mean & $\mathbf{6 6 . 9 9}$ & $\mathbf{6 8 . 2 8}$ & 60.91 & 63.03 \\
& Final & $\mathbf{9 2 . 3 9}$ & $\mathbf{9 2 . 3 3}$ & 89.50 & 86.90 \\
& Mean & 65.68 & 67.70 & $\mathbf{6 3 . 5 8}$ & $\mathbf{6 5 . 5 0}$ \\
& Final & 92.27 & 90.77 & $\mathbf{9 1 . 0 2}$ & 91.03 \\
& Mean & 65.80 & 61.34 & 59.06 & 60.30 \\
& Final & 92.12 & 85.06 & 89.77 & 86.61 \\
\hline
\end{tabular}

Table 2: Ablation study of $\lambda$ parameters on subset of SVHN dataset. Mean across epochs and accuracy(\%) of the Final model is reported.

metrics considered are: Euclidean $\left(\|u-v\|_{2}\right)$, Cosine (1 $\left.\frac{u \cdot v}{\|u\|_{2}\|v\|_{2}}\right)$, Correlation $\left(1-\frac{(u-\bar{u}) \cdot(v-\bar{v})}{\|(u-\bar{u})\|_{2}\|(v-\bar{v})\|_{2}}\right)$ where $\bar{u}$ is the mean elements of vector $u$ and Gaussian $\left(\exp -\frac{\left(\|u-v\|^{2}\right)}{2 \sigma^{2}}\right)$.

Figure 2(a) shows the result of the experiment. These results suggest that the Euclidean distance metric gives lower test error than the others.

\section{Effect of Learning Rate and Batch Size}

Batch size and learning rate are the most important hyperparameters that impact the learning dynamics of the model. In order to study the robustness of the model trained with submodular mini-batches, we vary batch size and learning rate, keeping all other parameters the same. We compare with SGD trained with the same set of hyper-parameters for a fair comparison.

Figure 2(c) reports the results when mini-batch sizes are set to 50,100 and 200. Our results agree with the common consensus [Li et al., 2014] that increasing the batch-size decreases the rate of convergence, still SMDL beats SGD by a consistent margin (solid lines in the graph) for all the batch sizes. We set the learning rate to $0.1,0.01$ and 0.001 and observe the same similar pattern in Figure 2(b). These results show that the performance of SMDL is robust to learning rate and batch size changes.

\section{Conclusion}

In this work, we cast the selection of diverse and informative mini-batches for training a deep learning model as a submodular optimization problem. We design a novel submodular objective and propose a scalable algorithm to do submodular selection. Extensive experimental valuation on three datasets reveals significant improvement in convergence and generalization performance of the model trained with submodular mini-batches over SGD and Loss based sampling [Loshchilov and Hutter, 2015]. The ablation results show that the method is robust to changes in batch size and learning rate.

\section{Acknowledgements}

We would like to thank MHRD, Govt of India for funding the personnel involved, Microsoft Research India and ACMIndia/IARCS for the travel grants to present the work and the anonymous reviewers for their valuable feedback. 


\section{References}

[Alain et al., 2015] Guillaume Alain, Alex Lamb, Chinnadhurai Sankar, Aaron Courville, and Yoshua Bengio. Variance reduction in sgd by distributed importance sampling. arXiv preprint arXiv:1511.06481, 2015.

[Allen-Zhu, 2017] Zeyuan Allen-Zhu. Katyusha: The first direct acceleration of stochastic gradient methods. The Journal of Machine Learning Research, 18(1):8194-8244, 2017.

[Brahma and Othon, 2018] Pratik Prabhanjan Brahma and Adrienne Othon. Subset replay based continual learning for scalable improvement of autonomous systems. In CVPR Workshops, pages 1179-11798. IEEE, 2018.

[Chakraborty et al., 2015] Shayok Chakraborty, Vineeth Balasubramanian, and Sethuraman Panchanathan. Adaptive batch mode active learning. IEEE TNNLS, 26(8):1747-1760, 2015.

[Chang et al., 2017] Haw-Shiuan Chang, Erik Learned-Miller, and Andrew McCallum. Active bias: Training more accurate neural networks by emphasizing high variance samples. In Advances in Neural Information Processing Systems, pages 1002-1012, 2017.

[Das and Kempe, 2008] Abhimanyu Das and David Kempe. Algorithms for subset selection in linear regression. In ACM STOC, pages 45-54. ACM, 2008.

[He et al., 2016] Kaiming He, Xiangyu Zhang, Shaoqing Ren, and Jian Sun. Deep residual learning for image recognition. In $C V P R$, pages 770-778, 2016.

[Ioffe and Szegedy, 2015] Sergey Ioffe and Christian Szegedy. Batch normalization: Accelerating deep network training by reducing internal covariate shift. arXiv preprint arXiv:1502.03167, 2015.

[Johnson and Zhang, 2013] Rie Johnson and Tong Zhang. Accelerating stochastic gradient descent using predictive variance reduction. In NIPS, pages 315-323, 2013.

[Katharopoulos and Fleuret, 2017] Angelos Katharopoulos and François Fleuret. Biased importance sampling for deep neural network training. arXiv preprint arXiv:1706.00043, 2017.

[Katharopoulos and Fleuret, 2018] Angelos Katharopoulos and François Fleuret. Not all samples are created equal: Deep learning with importance sampling. arXiv:1803.00942, 2018.

[Kingma and Ba, 2014] Diederik P Kingma and Jimmy Ba. Adam: A method for stochastic optimization. arXiv preprint arXiv:1412.6980, 2014.

[Krizhevsky and Hinton, 2009] Alex Krizhevsky and Geoffrey Hinton. Learning multiple layers of features from tiny images. Technical report, Citeseer, 2009.

[Li et al., 2014] Mu Li, Tong Zhang, Yuqiang Chen, and Alexander J Smola. Efficient mini-batch training for stochastic optimization. In Proceedings of the 20th ACM SIGKDD international conference on Knowledge discovery and data mining, pages 661670. ACM, 2014.

[Li et al., 2016] Chengtao Li, Stefanie Jegelka, and Suvrit Sra. Fast dpp sampling for nystr $\ "$ om with application to kernel methods. arXiv preprint arXiv:1603.06052, 2016.

[Lin and Bilmes, 2011] Hui Lin and Jeff Bilmes. A class of submodular functions for document summarization. In $A C L$, pages 510-520. Association for Computational Linguistics, 2011.

[Loshchilov and Hutter, 2015] Ilya Loshchilov and Frank Hutter. Online batch selection for faster training of neural networks. ICLR Workshops, 2015.
[Minoux, 1978] Michel Minoux. Accelerated greedy algorithms for maximizing submodular set functions. In Optimization techniques, pages 234-243. Springer, 1978.

[Mirzasoleiman et al., 2013] Baharan Mirzasoleiman, Amin Karbasi, Rik Sarkar, and Andreas Krause. Distributed submodular maximization: Identifying representative elements in massive data. In Advances in Neural Information Processing Systems, pages 2049-2057, 2013.

[Mirzasoleiman et al., 2015] Baharan Mirzasoleiman, Ashwinkumar Badanidiyuru, Amin Karbasi, Jan Vondrák, and Andreas Krause. Lazier than lazy greedy. In $A A A I, 2015$.

[Nemhauser et al., 1978] George L Nemhauser, Laurence A Wolsey, and Marshall L Fisher. An analysis of approximations for maximizing submodular set functions-i. Mathematical programming, 14(1):265-294, 1978.

[Netzer et al., 2011] Yuval Netzer, Tao Wang, Adam Coates, Alessandro Bissacco, Bo Wu, and Andrew Y Ng. Reading digits in natural images with unsupervised feature learning. In NIPS workshop, page 5, 2011.

[Paszke et al., 2017] Adam Paszke, Sam Gross, Soumith Chintala, Gregory Chanan, Edward Yang, Zachary DeVito, Zeming Lin, Alban Desmaison, Luca Antiga, and Adam Lerer. Automatic differentiation in pytorch. In NIPS-W, 2017.

[Singh and Balasubramanian, 2018] Krishna Kant Singh and Vineeth N Balasubramanian. Submodular importance sampling for neural network training. Master's thesis, Indian Institute of Technology Hyderabad, 2018.

[Thangarasa and Taylor, 2018] Vithursan Thangarasa and Graham W Taylor. Self-paced learning with adaptive deep visual embeddings. arXiv preprint arXiv:1807.09200, 2018.

[Wei et al., 2014] Kai Wei, Yuzong Liu, Katrin Kirchhoff, Chris Bartels, and Jeff Bilmes. Submodular subset selection for largescale speech training data. In ICASSP, pages 3311-3315. IEEE, 2014.

[Wei et al., 2015] Kai Wei, Rishabh Iyer, and Jeff Bilmes. Submodularity in data subset selection and active learning. In ICML, pages 1954-1963, 2015.

[Zhang et al., 2017] Cheng Zhang, Hedvig Kjellstrom, and Stephan Mandt. Determinantal point processes for mini-batch diversification. arXiv preprint arXiv:1705.00607, 2017.

[Zhang et al., 2018] Cheng Zhang, Cengiz Öztireli, Stephan Mandt, and Giampiero Salvi. Active mini-batch sampling using repulsive point processes. arXiv:1804.02772, 2018.

[Zhao and Zhang, 2014] Peilin Zhao and Tong Zhang. Accelerating minibatch stochastic gradient descent using stratified sampling. arXiv preprint arXiv: $1405.3080,2014$

[Zhao and Zhang, 2015] Peilin Zhao and Tong Zhang. Stochastic optimization with importance sampling for regularized loss minimization. In $I C M L$, pages 1-9, 2015.

[Zhou and Bilmes, 2018] Tianyi Zhou and Jeff Bilmes. Minimax curriculum learning: Machine teaching with desirable difficulties and scheduled diversity. In ICLR, 2018. 